\title{
The Problems and Countermeasures of the Current Situation of Sexual Health Education in Primary School Students in China
}

\author{
Yan Shunqin $^{1}$ \\ ${ }^{1}$ School of education, China West Normal University, nanchong, China \\ Correspondence: Yan Shunqin, School of education, China West Normal University, nanchong, China, ON., No 1 Shi \\ Da Road, Nanchong City, Sichuan Province, China.
}

Received: February 7, 2016

doi:10.11114/ijsss.v4i5.1527
Accepted: March 21, 2016

Available online: April 7, 2016

URL: http://dx.doi.org/10.11114/ijsss.v4i5.1527

\begin{abstract}
The sexual health education in primary school students, besides settling the sexual problems of students in primary school stage, is also the preparation for adolescent sexual health education, which will directly affect the sexual health level of adolescents and even adult individuals. Based on the review of the current situation of sexual health level of primary school students, this paper analyzes the major contradictions in the current primary school health education, and puts forward the strategies to promote the sexual health education in primary school students.
\end{abstract}

Keywords: China, primary school students, sexual health education, contradiction, strategy

\section{Introduction}

The hesitation of talking about "sex" in Chinese culture since ancient times caused the backwardness and ignorance of sexual health education in China. Because of that, the scientific sexual health education started late in China. Literature about sexual health education shows that the main objects of current sexual health education are adolescent secondary school students and post-adolescent college students, while there are relatively fewer researches on sexual health education in primary school students. It should be pointed out that the success of adolescent sexual health education, in fact, depends largely on the results of the sexual health education in primary school stage. Moreover, the earlier the sexual health education starts, the less will the students be influenced by wrong sexual information in the future, and the higher their sexual health level will be. Therefore, it is of great practical and social significance to carry out sexual health education scientifically and appropriately.

\subsection{The current situation of the sexual health level of primary school students in China}

Literatures show that currently the little sexual health education in primary school students is almost all given in senior grades (grade 5 and 6). And according to the research, the current sexual health level of primary school students in China is as follow.

1) Students know little about sex. There are many blind zones (Zhou Yuehong, 2011). They lack the correct cognition of simple knowledge of sex. For example, less than $60 \%$ students can answer correctly questions about sex like the knowledge of sexual organs, menstruation, and spermatorrhea (Yan Deyan, 2009). They lack enough basic knowledge of adolescent development, and know even less about reproductive organs with less than $30 \%$ correct answers about this in students (Wo Wenzhi \& Cheng Jing, 2014).

2) There is a low rate to accept the physical and psychological change in adolescence. About $50 \%$ girls and $40 \%$ boys will feel shy, nervous and even frightened when experiencing menarche and first spermatorrhea respectively. Only 50\% girls consider it normal to have menarche and know health care in periods and among the 52\% boys who view spermatorrhea as normal, about half have normal feeling after emission, and the other half think it will affect their health and make them feel guilty and burdened (Yan Deyan, 2009).

3) Students lack the knowledge to prevent venereal diseases like AIDS. $45 \%$ students don't know the transmission route and prevention of such venereal diseases as AIDS, and there is a low proportion of those who can answer accurately the detection of AIDS and whether it can be cured (Qi Lin, 2013).

4) Students have a weak awareness of self-protection of sex. Primary school students (especially those junior students 
who do not have basic knowledge of sexual psychology) are not quite aware and capable of telling what is and which behavior is sexual assault. Even though they can recognize it, they almost have no proper coping strategy or have difficulty in finding the one to help them. According to the inquiry of Chen Jingqi on the knowledge and skills of sexual assault prevention in primary school students, students of grade two in primary school lack the basic knowledge and skills of prevention of sexual assault to children （Chen Jingqi \& Kong Yanqiu \& Li Xiuzhen, 2012）.

5) The degree of openness of sexual concept is comparatively improved. Students are able to associate with the opposite sex moderately and communicate on topics about sex at ease (Zhou Yuehong, 2011). But some researches also show that some girls feel somewhat confused when associating with boys.

6) Although it is rare for primary school students to have difficulty in gender identification, there are still problems in it. Inquiries show that $4.41 \%$ boys and $1.54 \%$ girls do not like their own sex, and $2.94 \%$ boys and $3.08 \%$ girls choose to be the opposite sex if given the opportunity to choose. Also some inquiries show that $16 \%$ girls think boys are superior to girls and 5\% girls hope to become boys (Yan Deyan, 2009).

From the above, it can be seen that the overall sexual health level of primary school students is comparatively low at the present stage of China, mainly because of the following problems.

\section{The major contradictions of sexual health education in primary school students in current China.}

The author often goes to give lectures about adolescent sexual health education in primary and secondary schools, and often exchanges ideas and experiences about this with teachers and parents. According to these experiences and the results of the researches, it can be found that there are two major contradictions in the present primary school sexual health education in our country.

2.1 The contradiction between the great attention from national policy to primary school sexual health education and the difficulties in its implement in schools.

Issued in 2008 by the Ministry of Education, the Guidelines of Health Education in Primary and Secondary schools puts forward the goals and basic content of health education. It clearly points out that the sexual health education should be started in primary school stage, which is divided into three levels: level 1 (grade 1-2), level 2 (grade 3-4), and level 3 (grade 5-6). It also points out the content of sexual health education in various levels: level 1 (basic knowledge of birth and growth of life, and the knowledge of "where I come from"), level 2 (the life cycle of humans, a preliminary understanding of functions of children's and adolescents' main body organs, and how to protect themselves), level 3 (characteristics of adolescent development and differences in it between boys and girls, the menarche and its significance to girls, the first spermatorrhea and its significance to boys, the health care of mutation, personal hygiene knowledge in adolescence, etc.).However, the result of the implementation of sexual health education in primary school students is not optimistic. It can be seen that although great importance is attached by national policy to primary school sexual health education, there are difficulties in its implementation.

1) The first difficulty is the backward concept. At present, many school leaders in most ordinary primary schools are not aware enough of the importance of sexual health education. Some even worry that too early sexual education will have such side effect as making the children impure. Therefore, the school refuses all sexual health education. In some schools, in order not to disobey the policy, some courses about sexual education are set with professional teachers, but those courses were not really regularly and seriously given, because the focus of school education is still on academic scores and enrollment rate.

2) The second difficulty is the unqualified teaching staff. Teachers of sexual health education are highly required not only to have professional knowledge of psychology, sociology, medicine and law, but also to have open and healthy sexual concepts, and the ability to establish good relations with their students. This makes it difficult for many schools to smoothly carry out sexual health education. At present, teachers of sexual health education are mainly biological teachers, or moral education teachers, or psychological teachers if better. Such teachers, therefore, feel quite difficult in the teaching process.

3) The third difficulty is the lack of teaching materials, which is the most outstanding problem in sexual health education in current primary school stage. Teachers can't handle the width or the depth of their teaching without unified teaching materials that are suitable for various sexual health levels of primary school students.

\subsection{The contradiction between the gradually open sexual concept of parents and their low level and improper methods} in sexual health education

Most born in 1980s and with high education, the parents of primary school students at current stage of China have a gradually open sexual concept and no longer take "sex" as a kind of taboo. Nevertheless, although they are aware of the necessity of sexual health education to their children, the family sexual health education is not optimistic. The following 
are the reasons.

1) Parents don't have enough knowledge of sex. Since the parents didn't receive scientific and systematic sexual health education, when asked by their children questions about sex, few of them can answer reasonably. Thus they often avoid answering, cheat, restrain, blame, or give incomplete answers, which make the children confused and curious, suspicious and angry, disgusted and disappointed, self-abased and guilty, disordered and ignorant, and so on. In addition, many parents don't have a thorough understanding of sexual health education. They believe that the sexual health education to children is to let their kids recognize sexual organs, which is far from enough.

2) Parents don't have enough appropriate teaching methods. Family sexual health education needs opportunities and skills. So when many parents are doing this without any preparation, nor with any carries like pictures, photos or TV programs, their children will feel it very abrupt. Furthermore, before the questions about sex proposed unexpectedly by children in public, they don't know how to deal with the embarrassment and delay the satisfaction of answering, which often makes the children feel discouraged.

3) There is not clearly job division or enough cooperation between parents. In the special work of sexual health education, there should be proper job division and cooperation between parents. Especially with the growth of children, the parent of the same sex should be more involved in the child's sexual health education. But in China, mothers always play the leading role in family education. This situation makes the sexual health education to boys selectively ignored. Their acquirement of knowledge about sex relies more on the communication with friends, or on the information from books, magazines and the internet. Since most of them lack the ability to discriminate information of sex, they may easily go astray once obtaining some negative and obscene information.

\section{The strategies to improve the sexual health education level of primary school students in China}

At present, the overall sexual health level of primary school students in our country is generally low. Therefore, some practical strategies are necessary to promote the sexual health education in primary school students.

3.1 Schools should implement policies to effectively carry out systematic sexual health education, and to set appropriate content and ways of teaching in sexual health education according to different levels of students at primary school stage.

As the major channel of formal education, schools should start early and systematically sexual health education like the teaching of other courses. Meanwhile, schools should take into consideration the ability of primary school students to understand at different levels, what sex knowledge they already have, their possible sexual behaviors and sexual interests. In junior stage of primary school, the key points of sexual education includes the origin and birth of life, the cycle of life, organs of human body (reproductive organs), the private places of body and so on. In addition, appropriate sexual safety education is needed to help students to know the ways to protect the body from sexual harassment, the right to let their body privacies be respected and the duty to respect others. It is a golden period to carry out sexual education in junior grades in primary schools, for students at this stage will accept and absorb the knowledge of sex the same as that of other courses. Because of this, it is of vital importance for teachers to have scientific and realistic teaching attitude.

With the growth of age, the senior primary school students grow gradually from childhood to adolescence. Thus their individual psychology (especially sexual psychology) takes a significant change, and the sexual consciousness begins to wake up. On the basis of the mastery of sex knowledge, the focus of sexual health education at this stage lies in the attention to students' sexual psychology, which mainly includes: the proper attitude to the possible body changes in adolescence, adolescent health, the appreciation of one's own physical characteristics, self-identification of one's gender, gender equality, respect for the characteristics of the opposite sex, normal and healthy cross-gender intercommunication and the knowledge of its boundary, how to deal with unusual intercommunication (such as love letters, notes and dates), the knowledge of possible normal changes in psychology, and so on. In addition, children need to be taught to know how to protect themselves from sexual harassment or sexual assault, how to seek for help and protection if confronted with sexual assault, and so on. Due to the awakening of students' sexual consciousness, there should be breakthrough of conventional thinking in the classes of sexual education. For example, in the sexual health education of senior primary school students in Taiwan, positive sexual attitude can be cultivated by means of group psychological guidance (Feng Jiayu \& Yan Hanwen \& Gao Songjing, 2015).

3.2 It should be complementary between families, schools, and the society in sexual health education.

Family is the first place to provide children with sexual health education as well as the prelude of sexual health education in school. Children, when at very early age, will ask questions about sex and their views and attitudes toward sex, and their ways of exploring sex depend highly on the initial guidance of their parents. Family sexual health education is at the same time the extension of school sexual health education, for sexual health education is also life 
education, in which love and responsibility is the essence. The seed of school sexual health education can thrive with the nurture of family.

Research shows that the junior primary school students are more likely to ask questions about sex directly to their parents ( Li Shuangfei \& Ma Jia \& Sun Xiaomian, 2011). Although many parents have realized the role and significance of sexual health education, they feel it beyond their ability in the process of education because of the lack of knowledge of sex and educating approach. Therefore, besides the sexual health education courses, the schools should arrange related lectures for parents to enrich their knowledge and to guide them on how to talk with their children about sex. Only when there is a good cooperation between families and schools can a good environment be created for the healthy growth of children. In communities, relevant educating activities should be organized regularly to provide parents with the opportunities to learn and to exchange confusion and experience. In addition, the society should provide children with a healthy growing environment in such fields as guidance of public opinions and culture construction. Families, schools and the society, with good cooperation, should play a good role on their own in the education of primary school students at different ages.

\section{Conclusion}

It is of great significance to carry out sexual health education at primary school stage. The personality development of any individual will be affected if sexually unhealthy. Families, schools and the society should cooperate with each other in the sexual health education of primary school students, and carry out their duties to ensure the improvement of the sexual health level of primary school students in China.

\section{Acknowledgements}

This thesis is the research fruit of the project "The Problems and Countermeasures of the Current Situation of Sexual Health Education in Primary School Students", the research project of the key Research Centre of Philosophy and Social Science in Sichuan-Sichuan Education Development Research Centre (Num: CJF14011, Principal: Yan Shunqinn).

\section{References}

Chen, J. Q., Kong, Y. Q., \& Li, X. Z. (2012). Inquiry on the Knowledge and Skills of Sexual Assault Prevention in Students of Grade Two in Primary Schools [J]. Health Care in Chinese Schools, 33(1), 12-14.

Feng, J. Y, Yan, H. W., \& Gao, S. J. (2015). Analysis of the Results of Sexual Education Intervention in Remote Rural Areas of Taiwan-Taking National Middle School in Hualian County as an Example [J]. Journal of Sexology in Taiwan, 11(1), 99-113.

Li, S. F., Ma, J., \& Sun, X. M. (2011). Analysis of the Inquiry of Sexual Psychology and Related Behaviors of Primary School Students in Shenzhen City [J]. Southern China Preventive Medicine, 37(2), 43-45.

Qi, L. (2013). Preliminary Study on Sexual Education in Primary Schools [J]. Chinese Sexual Science, 9, 68-71.

Wo, W. Z., \& Cheng, J. (2014). Study on the Current Situation of Sexual Education in Primary School Students in Sichuan [J]. Journal of Chengdu Institute of Technology, 17(2), 85-88.

Yan, D. Y. (2009). On Sexual Education in Senior Students in Primary Schools, Thesis of Master's Degree, Central China Normal University.

Zhou, Y. H. (2011). Study on the Current Situation of Primary School Students and the Countermeasures of Sexual Education in Schools [J]. Journal of Changchun Normal University (Natural Science Edition), 30(6), 157-160.

\section{(cc) $\mathrm{Br}$}

This work is licensed under a Creative Commons Attribution 3.0 License. 